\title{
Potassium Hydroxide Impregnated Alumina (KOH-Alumina) as a Recyclable Catalyst for the Solvent-Free Multicomponent Synthesis of Highly Functionalized Substituted Pyridazines and/or Substituted Pyridazin-3(2H)-ones under Microwave Irradiation
}

\author{
Hormi Mecadon and Bekington Myrboh \\ Department of Chemistry, North-Eastern Hill University (NEHU), Permanent Campus, Umshing, Mawlai,
} Shillong 793022, Meghalaya, India

Correspondence should be addressed to Bekington Myrboh, bmyrboh@nehu.ac.in

Received 27 January 2011; Accepted 3 March 2011

Academic Editors: G. R. Cook, G. Kirsch, and J. Perez-Castells

Copyright ( $) 2011 \mathrm{H}$. Mecadon and B. Myrboh. This is an open access article distributed under the Creative Commons Attribution License, which permits unrestricted use, distribution, and reproduction in any medium, provided the original work is properly cited.

\begin{abstract}
The work described herein employs potassium hydroxide impregnated alumina (KOH-alumina) as a mild, efficient, and recyclable catalyst for a one-pot solvent-free and environmentally safer synthesis of 3,4,6-triarylpyridazines and some substituted pyridazines from active methylene carbonyl species, 1,2-dicarbonyls, and hydrazine hydrate by microwave (MW) irradiation. The method offers highly convergent, inexpensive, and functionality-tolerable procedure for rapid access to important pyridazine compounds in good yields.
\end{abstract}

\section{Introduction}

Pyridazines have received considerable attention because of their important pharmacological and biological properties [1]. Several pyridazine compounds exhibit antimicrobial [2], potent analgesic [3], COX inhibitor [4], antidiabetic [5], antihypertensive [6], herbicidal [7], anticancer [8], and antifungal [9] activities. Further, various pyridazinones have been used as intermediates for drugs and agrochemicals [5] and blood platelet aggregation inhibitors [10].

The synthesis of pyridazine frameworks has been achieved primarily by the addition of hydrazine or its derivative to an appropriate 1,4-diketones and 1,4-ketoacids [11-13]. Other various pyridazines particularly aminopyridazines have been prepared from polyfunctionalized nitriles, especially via the Jaap-Klingemaan reaction [14-18]. The literature also showed the preparation of pyridazines and pyridazinones involving active methylene species, benzil, and hydrazine. However, the methods employed harsh bases
[19-21] or acids [22] in presence of hazardous solvents, and also the reactions require long period of time to complete. Therefore, there is a need for developing a milder and safer solvent-free procedure for the synthesis of substituted pyridazines especially because of the rise in demand for environmentally benign organic synthesis.

To address the challenge of green synthesis, multicomponent reactions (MCRs) provide a solution since they are more efficient, cost effective, and less wasteful than traditional methods. Such synthetic approach, however, when teamed with microwave (MW) irradiation, facilitates the reaction better as MW gives very efficient thermal management and atom efficiency thus resulting in faster reaction with an increased product yield. In another development, in recent years, the use of inorganic solid supports as catalysts for the synthesis of various biologically active molecules has increased tremendously. Among these inorganic solid supports, potassium hydroxide coated with alumina $(\mathrm{KOH}-$ alumina) has been a versatile reagent for various reactions 


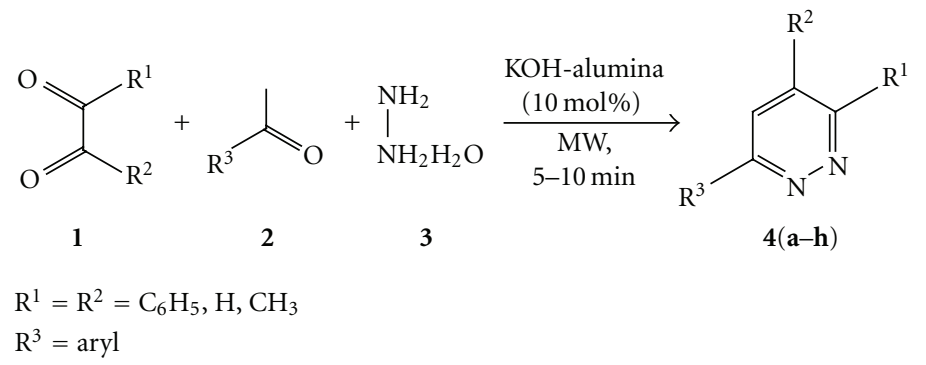

Scheme 1: One-pot synthesis of substituted pyridazines.

TABLE 1: Optimization of the reaction condition and the catalyst recyclability with compound $4 \mathbf{a}$.

\begin{tabular}{lccc}
\hline Entry & Reaction conditions & Time $(\min )$ & Yield $(\%)^{\mathrm{a}}$ \\
\hline 1 & KOH-alumina $(5 \mathrm{~mol} \%)$ & 3 & 57 \\
2 & KOH-alumina $(5 \mathrm{~mol} \%)$ & 10 & 64 \\
3 & KOH-alumina $(10 \mathrm{~mol} \%)$ & 2 & 72 \\
4 & KOH-alumina $(10 \mathrm{~mol} \%)$ & 4 & 89 \\
5 & KOH-alumina $(10 \mathrm{~mol} \%)$ & 6 & 88 \\
6 & KOH-alumina (10 mol\%) & 8 & 61 \\
7 & KOH-alumina (recycled once) & 15 & 30 \\
8 & KOH-alumina (recycled twice) & 18 & 22 \\
9 & KOH-alumina (recycled three times) & 22 & \\
\hline
\end{tabular}

${ }^{\mathrm{a}}$ Isolated yields.

and transformations such as in transesterification and biodiesel production [23-26], ester hydrolysis [27], selective alkylation [28-30], Michael addition [31], cyanoethylation [32], and gas phase dehydrogenations [33]. It has also been found that $\mathrm{KOH}$-alumina exhibited the highest basicity and superior catalytic activity among the alumina-supported alkaline catalysts during transesterification processes [25]. Moreover, $\mathrm{KOH}$-alumina can be prepared easily and is inexpensive. In view of these advantages in the applications of heterogeneous catalysts in the synthesis of heterocyclic compounds, we have chosen $\mathrm{KOH}$-alumina $(10 \%$ in alumina) for the synthesis of some substituted pyridazines.

Therefore, based on our previous work on pyridazine synthesis [21] and in conjunction with our current research aimed at development of synthetic methodologies using solid support catalysts through MCR's [34-36], we report herein the three-component neat synthesis of 3,4,5triarylpyridazines and other substituted pyridazines using $\mathrm{KOH}$-alumina $(10 \mathrm{~mol} \%)$ by the microwave irradiation technique (Scheme 1).

Initially, the three-component synthesis was optimized by irradiating a mixture of acetophenone $(2 \mathbf{a})(0.2 \mathrm{~mL}$, $1.50 \mathrm{mmol})$, benzil (1) $(0.32 \mathrm{~g}, 1.50 \mathrm{mmol})$ and hydrazine hydrate $(3)(0.10 \mathrm{~mL}, 2.00 \mathrm{mmol})$ in presence of $5 \mathrm{~mol} \%$ $\mathrm{KOH}$-alumina in a microwave reactor at $100^{\circ} \mathrm{C}$ for three minutes which afforded the product $4 \mathbf{a}$ in $57 \%$ yield. The same reaction when irradiated for ten minutes gave $\mathbf{4 a}$ in $64 \%$ yield. By varying the amount of the catalyst and irradiation time, optimization was finally arrived at $10 \mathrm{~mol} \%$ of $\mathrm{KOH}$-alumina which significantly resulted in $89 \%$ of the product $4 \mathrm{a}$ (Table 1). In another attempt, the catalyst recovered from the reaction after filtration, and washing with ethyl acetate was used further for the condensation of acetophenone (2a) $(0.20 \mathrm{~mL}, 1.50 \mathrm{mmol})$, benzil (1) $(0.32 \mathrm{~g}, 1.50 \mathrm{mmol})$, and hydrazine hydrate $(3)(0.10 \mathrm{~mL}$, $2.00 \mathrm{mmol}$ ). Interestingly, the reaction was observed to complete within 15 min of irradiation giving $4 \mathrm{a}$ in $61 \%$ yield. The results of the reactions using recycled $\mathrm{KOH}$-alumina are shown in Table 1.

Thus, the present method was employed for the synthesis of a series of 3,4,5-triarylpyridazines involving different aromatic ketones (4a-g, Table 2). Irrespective of the presence of different substituents in the ortho and para positions on the ring of various aromatic aldehydes, the reactions proceeded well to furnish the desired products in good yields (4a-g, Table 2). Unfortunately, the reaction performed with meta substituted aromatic ketones gave only unisolable intermediates and failed to furnish the desired products. On the other hand, polyaromatic acetophenones such as 2acetylnaphthalene $(\mathbf{2 h})$ underwent reaction smoothly with benzil (1) and hydrazine hydrate (3) to afford the desired product $\mathbf{4 h}$ in $77 \%$ yield (entry 9 , Table 2 ).

Similarly, the scope of this methodology was extended to synthesize other substituted pyridazines involving different active methylene carbonyl compounds such as ethyl cyano acetate $(\mathbf{5 a})$, diethyl malonate $(\mathbf{5 b})$, ethyl acetoacetate $(\mathbf{5} \mathbf{c})$, and acetyl acetone (5d) (Scheme 2). In all the cases the reactions proceeded fairly well and afforded the desired products in good yields $(\mathbf{6 a}-\mathbf{f})$, (Table 3$)$.

The reactions were clean and all the products were purified by simple work-up and crystallization except for products $4 \mathrm{~d}, 4 \mathrm{~g}$, $4 \mathrm{~h}$, and $6 \mathrm{~b}$ which were purified by column 


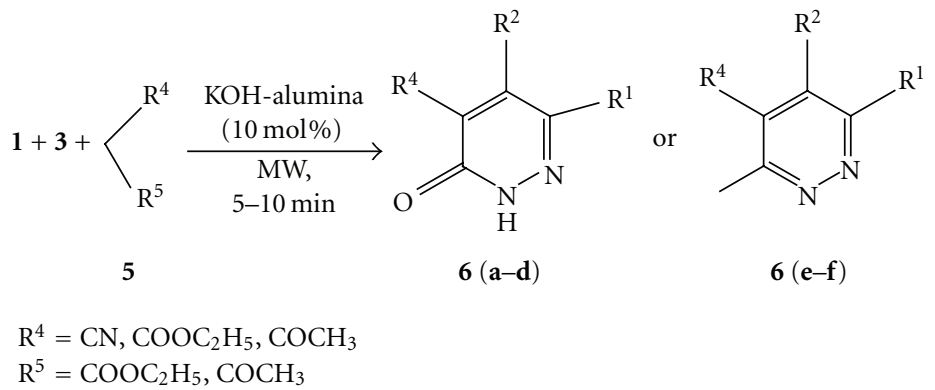

Scheme 2: One-pot synthesis of substituted pyridazines.

TABLE 2: KOH-alumina (10 mol\%) catalyzed solvent-free synthesis of substituted pyridazines under microwave (MW) irradiation.

\begin{tabular}{|c|c|c|c|c|c|c|}
\hline Entry & Substrate $\mathbf{2}\left(\mathrm{R}^{3}\right)$ & Substrate $1 \mathrm{R}^{1} / \mathrm{R}^{2}$ & Product 4 & Time $(\min )$ & Yield $(\%)^{\mathrm{a}}$ & M.p. $\left({ }^{\circ} \mathrm{C}\right)^{\mathrm{c}}$ \\
\hline 1 & $\mathrm{R}^{3}=\mathrm{C}_{6} \mathrm{H}_{5}$ & $\mathrm{R}^{1}=\mathrm{R}^{2}=\mathrm{C}_{6} \mathrm{H}_{5}$ & $4 a$ & 6 & 89 & $182-184^{[21]}$ \\
\hline 2 & $\mathrm{R}^{3}=4-\mathrm{CH}_{3} \mathrm{C}_{6} \mathrm{H}_{4}$ & $\mathrm{R}^{1}=\mathrm{R}^{2}=\mathrm{C}_{6} \mathrm{H}_{5}$ & $4 b$ & 5 & 86 & $160-162^{[21]}$ \\
\hline 3 & $\mathrm{R}^{3}=2-\mathrm{CH}_{3} \mathrm{OC}_{6} \mathrm{H}_{4}$ & $\mathrm{R}^{1}=\mathrm{R}^{2}=\mathrm{C}_{6} \mathrm{H}_{5}$ & $4 c$ & 10 & 76 & $137-139$ \\
\hline 4 & $\mathrm{R}^{3}=4-\mathrm{CH}_{3} \mathrm{OC}_{6} \mathrm{H}_{4}$ & $\mathrm{R}^{1}=\mathrm{R}^{2}=\mathrm{C}_{6} \mathrm{H}_{5}$ & $4 d$ & 8 & $85^{\mathrm{b}}$ & $164-166^{[21]}$ \\
\hline 5 & $\mathrm{R}^{3}=4-\mathrm{BrC}_{6} \mathrm{H}_{4}$ & $\mathrm{R}^{1}=\mathrm{R}^{2}=\mathrm{C}_{6} \mathrm{H}_{5}$ & $4 e$ & 8 & 81 & $147-149^{[21]}$ \\
\hline 6 & $\mathrm{R}^{3}=4-\mathrm{ClC}_{6} \mathrm{H}_{4}$ & $\mathrm{R}^{1}=\mathrm{R}^{2}=\mathrm{C}_{6} \mathrm{H}_{5}$ & $4 f$ & 6 & 76 & $179-181^{[21]}$ \\
\hline 7 & $\mathrm{R}^{3}=4-\mathrm{NO}_{2} \mathrm{C}_{6} \mathrm{H}_{4}$ & $\mathrm{R}^{1}=\mathrm{R}^{2}=\mathrm{C}_{6} \mathrm{H}_{5}$ & $4 g$ & 6 & $73^{\mathrm{b}}$ & $164-166^{[21]}$ \\
\hline 8 & $\mathrm{R}^{3}=$ & $\mathrm{R}^{1}=\mathrm{R}^{2}=\mathrm{C}_{6} \mathrm{H}_{5}$ & $4 h$ & 8 & $77^{\mathrm{b}}$ & $190-192^{[21]}$ \\
\hline
\end{tabular}

${ }^{a}$ Solated yield. ${ }^{b}$ Purified by column chromatography. ${ }^{c}$ Literature references.

chromatography using ethyl acetate and hexane. All the synthesized compounds were characterized by ${ }^{1} \mathrm{H} \mathrm{NMR},{ }^{13} \mathrm{C}$ NMR, IR, Mass, and Elemental analyses which were found to be in good agreement with the expected data.

From the mechanistic point of view, the formation of the triarlpyridazines $\mathbf{4}$ probably takes place through the addition of hydrazine hydrate to the 1, 4-dicarbonyl species (8) formed in situ by reaction between the acetophenone (2) and 1,2-dicarbonyl compound (1) in a similar fashion as reported earlier [21]. The overall plausible mechanism for the formation of the triarylpyridazines is depicted in Scheme 3.

\section{Conclusion}

In summary, we have established a mild and efficient method for the synthesis of highly functionalized substituted pyridazines and other substituted pyridazinones using $\mathrm{KOH}$ alumina $(10 \mathrm{~mol} \%)$. More importantly, the methodology presented here offers milder, more efficient, and particularly an environmentally friendly approach towards the synthesis of pyridazines by the use of potassium hydroxide impregnated on alumina as a recyclable catalyst.

\section{Experimental Section}

All the chemicals obtained commercially were directly used without further purification. $\mathrm{KOH}$-alumina was prepared according to the procedure reported by Sukata [28], however, as $10 \%$ of $\mathrm{KOH}$ adsorbed on neutral alumina. Melting points were recorded by open capillary tube method and were uncorrected. The thin layer chromatography was performed on ACME's silica or Merck precoated silica gel and the components were visualized in iodine chamber or by potassium permanganate spray technique. Flash column chromatography was performed on Merck silica gel (60-120 mesh) using ethyl acetate-hexane (3:7) as the eluent. IR spectra were recorded with Perkin-Elmer FT-IR spectrometer. The ${ }^{1} \mathrm{H}$ and ${ }^{13} \mathrm{C}$ NMR were recorded with Bruker AVANCE II 400 FT-NMR machine with TMS as the internal standard. Mass spectra were recorded with Waters ZQ-4000 equipped with ESI and APCI mass detector, and CHN was analyzed on Perkin-Elmer PE 2400 Series II.

\subsection{General Procedure}

3.1.1. Procedure for the Synthesis of $\mathbf{4}(\boldsymbol{a}-\boldsymbol{h})$. A thoroughly mixed aromatic ketone (2) (1.50 mmol), 1,2-dicarbonyl compound (1) (1.50 mmol), hydrazine hydrate (3) $(0.1 \mathrm{~mL}$, $2.00 \mathrm{mmol}$ ) in presence of $10 \mathrm{~mol} \% \mathrm{KOH}$-alumina was irradiated in a Chem Discover microwave reactor at $100^{\circ} \mathrm{C}$ (power $200 \mathrm{~W}$ ) at regular intervals of $60 \mathrm{sec}$ for 5-10 min. On completion of the reaction (monitored by thin layer chromatography), the reaction mixture was diluted with ethyl acetate and filtered on a sintered funnel. It was further washed down with ethyl acetate $(5 \mathrm{~mL} \times 4)$. The filtrate was then worked up with cold water, and the organic layer was separated and dried with anhydrous $\mathrm{Na}_{2} \mathrm{SO}_{4}$. The organic filtrate was evaporated in vacuo to afford the crude product 


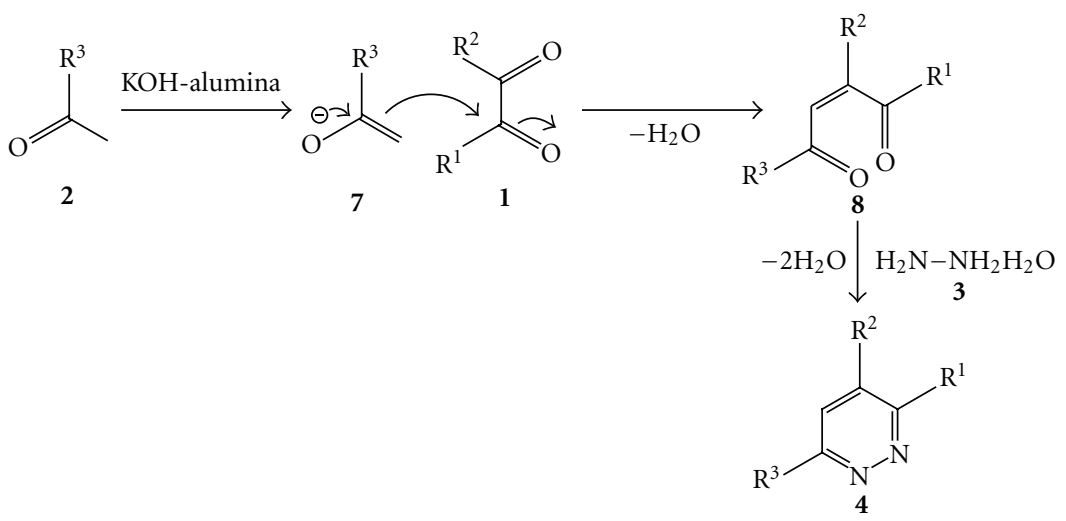

Scheme 3: Plausible mechanism for the formation of substituted pyridazines.

TABLE 3: KOH-alumina (10 mol\%) catalyzed solvent-free synthesis of substituted pyridazines under microwave (MW) irradiation.

\begin{tabular}{|c|c|c|c|c|c|c|}
\hline Entry & Substrate $\mathbf{5}\left(\mathrm{R}^{4}, \mathrm{R}^{5}\right)$ & Substrate $1 \mathrm{R}^{1} / \mathrm{R}^{2}$ & Product 6 & Time $(\min )$ & Yield $(\%)^{\mathrm{a}}$ & M.p. $\left({ }^{\circ} \mathrm{C}\right)^{\mathrm{c}}$ \\
\hline 1 & $\begin{array}{c}\mathrm{R}^{4}=\mathrm{CN} \\
\mathrm{R}^{5}=\mathrm{COOC}_{2} \mathrm{H}_{5}(\mathbf{5 a})\end{array}$ & $\mathrm{R}^{1}=\mathrm{R}^{2}=\mathrm{C}_{6} \mathrm{H}_{5}$ & $6 a$ & 8 & 81 & $270-272^{[19]}$ \\
\hline 2 & $(5 \mathbf{a})$ & $\mathrm{R}^{1}=\mathrm{R}^{2}=\mathrm{H}$ & $6 b$ & 8 & $76^{\mathrm{b}}$ & $182-184^{[19]}$ \\
\hline 3 & $(5 a)$ & $\mathrm{R}^{1}=\mathrm{R}^{2}=\mathrm{CH}_{3}$ & $6 c$ & 8 & 87 & $209-211^{[19]}$ \\
\hline 4 & $\mathrm{R}^{4}=\mathrm{R}^{5}=\mathrm{COOC}_{2} \mathrm{H}_{5}(\mathbf{5 b})$ & $\mathrm{R}^{1}=\mathrm{R}^{2}=\mathrm{C}_{6} \mathrm{H}_{5}$ & $6 d$ & 8 & 82 & $217-219^{[19]}$ \\
\hline 5 & $\begin{array}{c}\mathrm{R}^{4}=\mathrm{COOC}_{2} \mathrm{H}_{5} \\
\mathrm{R}^{5}=\mathrm{COCH}_{3}(5 \mathrm{c})\end{array}$ & $\mathrm{R}^{1}=\mathrm{R}^{2}=\mathrm{C}_{6} \mathrm{H}_{5}$ & $6 e$ & 8 & 86 & $77-79^{[20]}$ \\
\hline 6 & $\mathrm{R}^{4}=\mathrm{R}^{5}=\mathrm{COCH}_{3}(\mathbf{5 d})$ & $\mathrm{R}^{1}=\mathrm{R}^{2}=\mathrm{C}_{6} \mathrm{H}_{5}$ & $6 f$ & 8 & 79 & $132-134^{[20]}$ \\
\hline
\end{tabular}

Isolated yield. ${ }^{\mathrm{b}}$ Purified by column chromatography. ${ }^{\mathrm{c}}$ Literature references.

which was crystallized from ethanol (4a, 4b, 4c, 4e, and 4f) or purified by flash column chromatography $(\mathbf{4 d}, \mathbf{4 g}$, and 4h) over silica gel (60-120 mesh) using ethyl acetate-hexane $(3: 7)$ as the eluent to afford the 3,4,6-triarylpyridazines.

3.1.2. Procedure for the Synthesis of $\mathbf{6}(\boldsymbol{a}-\boldsymbol{f})$. A thoroughly mixed 1,2-dicarbonyl compound (1) (1.50 mmol) and hydrazine hydrate $(3)(2.00 \mathrm{mmol})$ was irradiated in a Chem Discover microwave reactor at $100^{\circ} \mathrm{C}$ (power $200 \mathrm{~W}$ ) for 5 minutes. The mixture was cooled and then introduced therein the active methylene species (5) $(1.50 \mathrm{mmol})$ and $\mathrm{KOH}$-alumina $(10 \mathrm{~mol} \%)$. The components were mixed thoroughly and subjected to microwave irradiation at $100^{\circ} \mathrm{C}$ (power 200 W) for 3-6 minutes. On completion of the reaction (monitored by thin layer chromatography), the reaction mixture was diluted with ethyl acetate and filtered on a sintered funnel. It was further washed down with ethyl acetate $(5 \mathrm{~mL} \times 4)$. The filtrate was then worked up with cold water and the organic layer was separated and dried with anhydrous $\mathrm{Na}_{2} \mathrm{SO}_{4}$. The organic filtrate was evaporated in vacuo to afford the crude product which was crystallized from ethanol $(\mathbf{6 a}, \mathbf{6 c}, \mathbf{6 d}, \mathbf{6 e}$, and $\mathbf{6 f})$ or purified by flash column chromatography (6b) over silica gel (60-120 mesh) using ethyl acetate-hexane $(3: 7)$ as the eluent to afford the pure product.

3,4,6-triphenylpyridazine (4a, Table 2). White solid; mp 182$184^{\circ} \mathrm{C} ;{ }^{1} \mathrm{H}$ NMR $\left(400 \mathrm{MHz}, \mathrm{CDCl}_{3}\right): \delta_{\mathrm{H}} 7.27-7.89(\mathrm{~m}, 13 \mathrm{H}$,
Ar-H), 8.20 (s, 1H, Ar-H), 8.20 (d, $2 \mathrm{H}, J=6.8 \mathrm{~Hz}, \mathrm{Ar}-\mathrm{H})$ ppm; ${ }^{13} \mathrm{C} \mathrm{NMR}\left(100 \mathrm{MHz}, \mathrm{CDCl}_{3}\right): \delta_{\mathrm{C}} 124.5,126.6,127.6$, $128.3,128.6,129.5,129.6,135.4,136.0,136.6,139.2,157.2$, $157.7 \mathrm{ppm}$; IR $(\mathrm{KBr}): \nu_{\max } 1075,1177,1394,1444,1488$, 1582, 2854, 2924, $3063 \mathrm{~cm}^{-1}$; MS $\left(\mathrm{ES}^{+}\right)$for $\mathrm{C}_{22} \mathrm{H}_{16} \mathrm{~N}_{2} 308.1$ found $308.9(\mathrm{M}+\mathrm{H})^{+}, 331.0(\mathrm{M}+\mathrm{Na})^{+}$; $\mathrm{CHN}$ calcd. for $\mathrm{C}_{22} \mathrm{H}_{16} \mathrm{~N}_{2} \mathrm{C}, 85.69 ; \mathrm{H}, 5.23 ; \mathrm{N}, 9.08$ found $\mathrm{C}, 85.71 ; \mathrm{H}, 5.38$; $\mathrm{N}, 9.32$.

3,4-diphenyl-6-p-tolylpyridazine (4b, Table 2). Light yellow solid; mp $160-162^{\circ} \mathrm{C} ;{ }^{1} \mathrm{H}$ NMR $\left(400 \mathrm{MHz} \mathrm{CDCl}_{3}\right): \delta_{\mathrm{H}} 2.43$ $\left(\mathrm{s}, 3 \mathrm{H}, \mathrm{CH}_{3}\right), 7.25-7.35(\mathrm{~m}, 10 \mathrm{H}, \mathrm{Ar}-\mathrm{H}), 7.48(\mathrm{~d}, 2 \mathrm{H}, \mathrm{J}=$ $6.8 \mathrm{~Hz}, \mathrm{Ar}-\mathrm{H}), 7.83$ (s, 1H, Ar-H), 8.08 (d, $2 \mathrm{H}, J=8.0 \mathrm{~Hz}$, Ar-H) ppm; ${ }^{13} \mathrm{C}$ NMR $\left(100 \mathrm{MHz}, \mathrm{CDCl}_{3}\right): \delta_{\mathrm{C}} 21.4,124.7$, $126.9,128.1,128.7,129.0,129.8,130.0,133.0,136.5,137.1$, $139.6,140.4,157.6,157.9 \mathrm{ppm}$; IR (KBr): $\nu_{\max } 1079,1222$, 1367, 1419, 1592, 2867, 2923, $3019 \mathrm{~cm}^{-1}$; MS $\left(\mathrm{ES}^{+}\right)$for $\mathrm{C}_{23} \mathrm{H}_{18} \mathrm{~N}_{2} 322.1$ found $323.0(\mathrm{M}+\mathrm{H})^{+}, 345.0(\mathrm{M}+\mathrm{Na})^{+}$; CHN calcd. for $\mathrm{C}_{23} \mathrm{H}_{18} \mathrm{~N}_{2} \mathrm{C}, 85.68 ; \mathrm{H}, 5.63 ; \mathrm{N}, 8.69$ found C,85.55; H, 5.65; N, 8.44.

6-(2-methoxyphenyl)-3,4-diphenylpyridazine (4c, Table 2). White solid; mp $137-139^{\circ} \mathrm{C}$; ${ }^{1} \mathrm{H}$ NMR $\left(400 \mathrm{MHz}, \mathrm{CDCl}_{3}\right)$ : $\delta_{\mathrm{H}} 3.85\left(\mathrm{~s}, 3 \mathrm{H}, \mathrm{OCH}_{3}\right), 7.00-7.30(\mathrm{~m}, 12 \mathrm{H}, \mathrm{Ar}-\mathrm{H}), 7.43(\mathrm{~s}$, $1 \mathrm{H}, \mathrm{Ar}-\mathrm{H}), 8.03(\mathrm{~d}, 2 \mathrm{H}, J=7.2 \mathrm{~Hz}, \mathrm{Ar}-\mathrm{H}) \mathrm{ppm} ;{ }^{13} \mathrm{C} \mathrm{NMR}$ $\left(100 \mathrm{MHz}, \mathrm{CDCl}_{3}\right): \delta_{\mathrm{C}} 55.8,111.4,121.4,124.7,128.2,128.7$, $128.9,129.2,130.1,131.5,131.6,136.3,137.0,139.1,156.9$, 157.3, 157.7 ppm; IR (KBr): $\nu_{\max } 1076,1199,1206,1371$, 
$1496,1509,2877,2943,3016 \mathrm{~cm}^{-1}$; $\mathrm{MS}\left(\mathrm{ES}^{+}\right)$for $\mathrm{C}_{23} \mathrm{H}_{18} \mathrm{~N}_{2} \mathrm{O}$ 338.1 found $339.0(\mathrm{M}+\mathrm{H})^{+}, 361.0(\mathrm{M}+\mathrm{Na})^{+}$; CHN calcd. for $\mathrm{C}_{23} \mathrm{H}_{18} \mathrm{~N}_{2} \mathrm{O}$ C, $81.63 ; \mathrm{H}, 5.36 ; \mathrm{N}, 8.28$ found $\mathrm{C}, 81.59 ; \mathrm{H}$, $5.16 ; \mathrm{N}, 8.35$.

6-(4-methoxyphenyl)-3,4-diphenylpyridazine (4d, Table 2). Yellow solid; mp $164-166^{\circ} \mathrm{C} ;{ }^{1} \mathrm{H}$ NMR $\left(400 \mathrm{MHz}, \mathrm{CDCl}_{3}\right)$ : $\delta_{\mathrm{H}} 3.87\left(\mathrm{~s}, 3 \mathrm{H}, \mathrm{OCH}_{3}\right), 7.04(\mathrm{~d}, 2 \mathrm{H}, J=8.8 \mathrm{~Hz}, \mathrm{Ar}-\mathrm{H}), 7.21-$ 7.35 (m, 8H, Ar-H), 7.47 (d, 2H, J = 6.8 Hz, Ar-H), 7.79 (s, $1 \mathrm{H}, \mathrm{Ar}-\mathrm{H}), 8.15$ (d, $2 \mathrm{H}, J=8.4 \mathrm{~Hz}, \mathrm{Ar}-\mathrm{H}) \mathrm{ppm} ;{ }^{13} \mathrm{C} \mathrm{NMR}$ $\left(100 \mathrm{MHz}, \mathrm{CDCl}_{3}\right): \delta_{\mathrm{C}} 55.39,114.4,124.2,128.2,128.3$, $128.4,128.6,128.7,129.0,130.0,136.7,137.2,139.5,157.2$, 157.6, 161.3 ppm; IR (KBr): $\nu_{\max } 1067,1208,1310,1487$, $1562,2877,2931,3012 \mathrm{~cm}^{-1}$; MS (ES ${ }^{+}$) for $\mathrm{C}_{23} \mathrm{H}_{18} \mathrm{~N}_{2} \mathrm{O} 338.1$ found $339.0(\mathrm{M}+\mathrm{H})^{+}, 361.0(\mathrm{M}+\mathrm{Na})^{+}$; $\mathrm{CHN}$ calcd. for $\mathrm{C}_{23} \mathrm{H}_{18} \mathrm{~N}_{2} \mathrm{O}$ C, 81.63; H, 5.36; N, 8.28 found C, 81.58; $\mathrm{H}$, $5.29 ; \mathrm{N}, 8.11$.

6-(4-bromophenyl)-3,4-diphenylpyridazine (4e, Table 2). White solid; mp $147-149^{\circ} \mathrm{C} ;{ }^{1} \mathrm{H}$ NMR (400 MHz, $\left.\mathrm{CDCl}_{3}\right)$ : $\delta_{\mathrm{H}}$ 7.05-7.45 (m, 12H, Ar-H), $7.74(\mathrm{~s}, 1 \mathrm{H}, \mathrm{Ar}-\mathrm{H}), 8.08$ $(\mathrm{d}, 2 \mathrm{H}, J=6.8 \mathrm{~Hz}) \mathrm{ppm} ;{ }^{13} \mathrm{C} \mathrm{NMR}\left(100 \mathrm{MHz}, \mathrm{CDCl}_{3}\right)$ : $\delta_{\mathrm{C}} 122.6,125.2,126.9,127.2,128.2,128.8,128.9,129.1$, $130.1,130.2,131.6,131.8,135.8,136.4,137.0,139.9$, $157.7,158.2 \mathrm{ppm}$; IR (KBr): $\nu_{\max } 1075,1296,1400,1488$, 1571, 2934, $3011 \mathrm{~cm}^{-1}$; MS $\left(\mathrm{ES}^{+}\right)$for $\mathrm{C}_{22} \mathrm{H}_{15} \mathrm{BrN}_{2} 386.0$ found $387.0(\mathrm{M}+\mathrm{H})^{+}, 409.0(\mathrm{M}+\mathrm{Na})^{+}$; CHN calcd. for $\mathrm{C}_{22} \mathrm{H}_{15} \mathrm{BrN}_{2} \mathrm{C}, 68.23 ; \mathrm{H}, 3.90 ; \mathrm{N}, 7.23$ found $\mathrm{C}, 68.21 ; \mathrm{H}$, $3.73 ; \mathrm{N}, 7.11$.

6-(4-chlorophenyl)-3,4-diphenylpyridazine (4f, Table 2). Light yellow solid; mp $179-181^{\circ} \mathrm{C}$; ${ }^{1} \mathrm{H} \mathrm{NMR}\left(400 \mathrm{MHz}, \mathrm{CDCl}_{3}\right)$ : $\delta_{\mathrm{H}}$ 7.06-7.44 (m, 12H, Ar-H), 7.73 (s, 1H, Ar-H), 8.08 $(\mathrm{d}, 2 \mathrm{H}, J=7.6 \mathrm{~Hz}) \mathrm{ppm} ;{ }^{13} \mathrm{C} \mathrm{NMR}\left(100 \mathrm{MHz}, \mathrm{CDCl}_{3}\right)$ : $\delta_{\mathrm{C}} 125.1,126.4,126.5,127.1,127.2,128.2,128.6,128.9$, 129.1, 129.5, 129.7, 130.1, 130.2, 135.9, 136.5, 139.8, 157.7, $158.2 \mathrm{ppm}$; IR (KBr): $\nu_{\max } 1076,1200,1301,1481,1546$, 2879, 2932, $3088 \mathrm{~cm}^{-1}$; MS $\left(\mathrm{ES}^{+}\right)$for $\mathrm{C}_{22} \mathrm{H}_{15} \mathrm{ClN}_{2} 342.1$ found $343.0(\mathrm{M}+\mathrm{H})^{+}, 365.0(\mathrm{M}+\mathrm{Na})^{+}$; $\mathrm{CHN}$ calcd. for $\mathrm{C}_{22} \mathrm{H}_{15} \mathrm{ClN}_{2} \mathrm{C}, 77.08 ; \mathrm{H}, 4.41 ; \mathrm{N}, 8.17$ found $\mathrm{C}, 77.26 ; \mathrm{H}$, $4.59 ; \mathrm{N}, 8.06$.

6-(4-nitrophenyl)-3,4-diphenylpyridazine (4g, Table 2). White solid; mp $164-166^{\circ} \mathrm{C} ;{ }^{1} \mathrm{H} \mathrm{NMR}\left(400 \mathrm{MHz}, \mathrm{CDCl}_{3}\right): \delta_{\mathrm{H}} 7.16-$ $7.44(\mathrm{~m}, 11 \mathrm{H}, \mathrm{Ar}-\mathrm{H}), 7.56$ (t, 1H, J = 7.2 Hz, Ar-H), 7.79 (s, $1 \mathrm{H}, \mathrm{Ar}-\mathrm{H}), 8.07$ (d, $2 \mathrm{H}, J=8.0 \mathrm{~Hz}, \mathrm{Ar}-\mathrm{H}) \mathrm{ppm} ;{ }^{13} \mathrm{C} \mathrm{NMR}$ $\left(100 \mathrm{MHz} \mathrm{CDCl}_{3}\right): \delta_{\mathrm{C}} 123.0,123.6,126.1,127.3,128.2$, $128.9,129.0,129.1,129.3,129.6,134.4,135.6,140.9,148.1$, $157.6,158.1 \mathrm{ppm}$; IR (KBr): $\nu_{\max } 1076,1230,1309,1441$, 1541, 2877, 2932, $3081 \mathrm{~cm}^{-1}$; MS $\left(\mathrm{ES}^{+}\right)$for $\mathrm{C}_{22} \mathrm{H}_{15} \mathrm{~N}_{3} \mathrm{O}_{2}$ 353.1 found $354.0(\mathrm{M}+\mathrm{H})^{+}, 376.0(\mathrm{M}+\mathrm{Na})^{+}$; CHN calcd. for $\mathrm{C}_{22} \mathrm{H}_{15} \mathrm{~N}_{3} \mathrm{O}_{2} \mathrm{C}, 74.78 ; \mathrm{H}, 4.28 ; \mathrm{N}, 11.89$ found $\mathrm{C}, 74.53$; $\mathrm{H}, 4.40 ; \mathrm{N}, 11.76$.

6-(naphthalen-2-yl)-3,4-diphenylpyridazine (4h, Table 2). White solid; mp $190-192{ }^{\circ} \mathrm{C} ;{ }^{1} \mathrm{H}$ NMR (400 MHz, $\left.\mathrm{CDCl}_{3}\right)$ : $\delta_{\mathrm{H}} 7.10-7.73(\mathrm{~m}, 18 \mathrm{H}, \mathrm{Ar}-\mathrm{H}) \mathrm{ppm} ;{ }^{13} \mathrm{C} \mathrm{NMR}(100 \mathrm{MHz}$, $\left.\mathrm{CDCl}_{3}\right): \delta_{\mathrm{c}} 124.2,125.4,125.8,126.1,127.3,127.9,128.3$,
$128.8,129.0,129.1,130.2,130.3,133.1,135.6,136.9,157.7$, $158.2 \mathrm{ppm}$; IR (KBr): $\nu_{\max } 1080,1234,1290,1438,1521$, 2861, 2932, 2995, $3043 \mathrm{~cm}^{-1}$; MS (ES ${ }^{+}$) for $\mathrm{C}_{26} \mathrm{H}_{18} \mathrm{~N}_{2} 358.1$ found $359.0(\mathrm{M}+\mathrm{H})^{+}, 381.0(\mathrm{M}+\mathrm{Na})^{+}$; $\mathrm{CHN}$ calcd. for $\mathrm{C}_{26} \mathrm{H}_{18} \mathrm{~N}_{2}$ C, 87.12; H, 5.06; N, 7.82 found C, 87.31; H, 5.21; $\mathrm{N}, 7.71$.

2,3-dihydro-3-oxo-5,6-diphenylpyridazine-4-carbonitrile ( $6 \mathbf{a}$, Table 3). White solid; mp $270-272{ }^{\circ} \mathrm{C} ;{ }^{1} \mathrm{H}$ NMR $(400 \mathrm{MHz}$, $\left.\mathrm{CDCl}_{3}+\mathrm{DMSO}-d_{6}\right): \delta_{\mathrm{H}} 6.91-7.94(\mathrm{~m}, 10 \mathrm{H}, \mathrm{Ar}-\mathrm{H}), 11.58$ (s, $1 \mathrm{H}, \mathrm{NH}) \mathrm{ppm} ;{ }^{13} \mathrm{C} \mathrm{NMR}\left(100 \mathrm{MHz}, \mathrm{CDCl}_{3}+\mathrm{DMSO}-\right.$ $\left.d_{6}\right): \delta_{\mathrm{C}} 110.3,116.5,127.8,128.9,129.1,129.6,130.2,131.4$, $132.7,133.6,135.3,146.9,158.2 \mathrm{ppm}$; IR (KBr): $v_{\max } 1010$, 1089, 1210, 1464, 1511, 1693, 2256, 2868, 2932, $3412 \mathrm{~cm}^{-1}$; MS $\left(\mathrm{ES}^{+}\right)$for $\mathrm{C}_{17} \mathrm{H}_{11} \mathrm{~N}_{3} \mathrm{O} 273.1$ found $274.0(\mathrm{M}+\mathrm{H})^{+}, 296.0$ $(\mathrm{M}+\mathrm{Na})^{+}$; $\mathrm{CHN}$ calcd. for $\mathrm{C}_{17} \mathrm{H}_{11} \mathrm{~N}_{3} \mathrm{O} \mathrm{C}, 74.71 ; \mathrm{H}, 4.06 ; \mathrm{N}$, 15.38 found $\mathrm{C}, 74.86 ; \mathrm{H}, 4.21 ; \mathrm{N}, 15.12$.

2,3-dihydro-3-oxopyridazine-4-carbonitrile (6b, Table 3). White solid; mp $182-184^{\circ} \mathrm{C}$; ${ }^{1} \mathrm{H}$ NMR $\left(400 \mathrm{MHz}, \mathrm{CDCl}_{3}\right.$ + DMSO- $\left.d_{6}\right): \delta_{\mathrm{H}} 7.24(\mathrm{~s}, 1 \mathrm{H}, \mathrm{Ar}-\mathrm{H}), 7.63(\mathrm{~s}, 1 \mathrm{H}, \mathrm{Ar}-\mathrm{H})$, $11.57(\mathrm{~s}, 1 \mathrm{H}, \mathrm{NH}) \mathrm{ppm} ;{ }^{13} \mathrm{C} \mathrm{NMR}\left(100 \mathrm{MHz}, \mathrm{CDCl}_{3}+\right.$ DMSO- $\left.d_{6}\right): \delta_{\mathrm{C}} 110.4,118.3,138.7,152.3,168.8 \mathrm{ppm} ; \mathrm{IR}$ (KBr): $\nu_{\max } 1089,1212,1400,1526,1676,2247,2881,2932$, $3310 \mathrm{~cm}^{-1}$; MS $\left(\mathrm{ES}^{+}\right)$for $\mathrm{C}_{5} \mathrm{H}_{3} \mathrm{~N}_{3} \mathrm{O} 121.0$ found $122.0(\mathrm{M}+$ $\mathrm{H})^{+}, 144.0(\mathrm{M}+\mathrm{Na})^{+}$; CHN calcd. for $\mathrm{C}_{5} \mathrm{H}_{3} \mathrm{~N}_{3} \mathrm{O} \mathrm{C}, 49.59$; $\mathrm{H}, 2.50 ; \mathrm{N}, 34.70$ found $\mathrm{C}, 49.53 ; \mathrm{H}, 2.41 ; \mathrm{N}, 34.62$.

2,3-dihydro-5,6-dimethyl-3-oxopyridazine-4-carbonitrile (6c, Table 3). White solid; mp $209-211^{\circ} \mathrm{C} ;{ }^{1} \mathrm{H}$ NMR $(400 \mathrm{MHz}$, $\left.\mathrm{CDCl}_{3}+\mathrm{DMSO}-d_{6}\right): \delta_{\mathrm{H}} 2.33\left(\mathrm{~s}, 3 \mathrm{H}, \mathrm{CH}_{3}\right), 2.50(\mathrm{~s}, 3 \mathrm{H}$, $\left.\mathrm{CH}_{3}\right), 11.25$ (s, 1H, NH) ppm; ${ }^{13} \mathrm{C} \mathrm{NMR}\left(100 \mathrm{MHz}, \mathrm{CDCl}_{3}\right.$ + DMSO- $\left.d_{6}\right): \delta_{\mathrm{C}} 9.9,27.6,116.7,126.2,152.8,157.4$, $167.9 \mathrm{ppm}$; IR (KBr): $\nu_{\max } 1046,1287,1412,1547,1671$, 2219, 2931, $3349 \mathrm{~cm}^{-1}$; MS $\left(\mathrm{ES}^{+}\right)$for $\mathrm{C}_{7} \mathrm{H}_{7} \mathrm{~N}_{3} \mathrm{O} 149.1$ found $150.0(\mathrm{M}+\mathrm{H})^{+}, 172.0(\mathrm{M}+\mathrm{Na})^{+}$; CHN calcd. for $\mathrm{C}_{7} \mathrm{H}_{7} \mathrm{~N}_{3} \mathrm{O}$ C, 56.37; H, 4.73; N, 28.17 found C, 56.21; H, 4.68; N, 28.32.

Ethyl 2,3-dihydro-3-oxo-5,6-diphenylpyridazine-4-carboxylate (6d, Table 3). White solid; mp $217-219^{\circ} \mathrm{C} ;{ }^{1} \mathrm{H}$ NMR $\left(400 \mathrm{MHz}, \mathrm{CDCl}_{3}\right): \delta_{\mathrm{H}} 0.90\left(\mathrm{t}, 3 \mathrm{H}, J=7.2 \mathrm{~Hz}, \mathrm{CH}_{3}\right), 4.05$ (q, $\left.2 \mathrm{H}, J=7.2 \mathrm{~Hz}, \mathrm{CH}_{2}\right), 7.03-7.27(\mathrm{~m}, 10 \mathrm{H}, \mathrm{Ar}-\mathrm{H}), 12.56$ $(\mathrm{s}, 1 \mathrm{H}, \mathrm{NH}) \mathrm{ppm} ;{ }^{13} \mathrm{C} \mathrm{NMR}\left(100 \mathrm{MHz}, \mathrm{CDCl}_{3}\right): \delta_{\mathrm{C}} 13.7$, $62.0,128.0,128.3,128.7,129.1,129.2,133.7,133.8,134.7$, $143.3,147.7,158.7,163.6 \mathrm{ppm}$; IR (KBr): $\nu_{\max } 1101,1200$, $1432,1500,1672,1768,2867,2931,3401 \mathrm{~cm}^{-1}$; MS $\left(\mathrm{ES}^{+}\right)$for $\mathrm{C}_{19} \mathrm{H}_{16} \mathrm{~N}_{2} \mathrm{O}_{3} 320.1$ found $321.0(\mathrm{M}+\mathrm{H})^{+}, 343.0(\mathrm{M}+\mathrm{Na})^{+}$; CHN calcd. for $\mathrm{C}_{19} \mathrm{H}_{16} \mathrm{~N}_{2} \mathrm{O}_{3} \mathrm{C}, 71.24 ; \mathrm{H}, 5.03 ; \mathrm{N}, 8.74$ found C, $71.43 ; \mathrm{H}, 5.17 ; \mathrm{N}, 8.68$.

Ethyl 3-methyl-5,6-diphenylpyridazine-4-carboxylate (6e, Table 3). White solid; mp $77-79^{\circ} \mathrm{C} ;{ }^{1} \mathrm{H}$ NMR $(400 \mathrm{MHz}$, $\left.\mathrm{CDCl}_{3}+\mathrm{DMSO}-d_{6}\right): \delta_{\mathrm{H}} 0.95\left(\mathrm{t}, 3 \mathrm{H}, J=7.4 \mathrm{~Hz}, \mathrm{CH}_{3}\right), 2.65$ $\left(\mathrm{s}, 3 \mathrm{H}, \mathrm{CH}_{3}\right), 4.05\left(\mathrm{q}, 2 \mathrm{H}, \mathrm{J}=7.6 \mathrm{~Hz}, \mathrm{CH}_{2}\right), 7.14-7.46(\mathrm{~m}$, $10 \mathrm{H}, \mathrm{Ar}-\mathrm{H}) \mathrm{ppm} ;{ }^{13} \mathrm{C} \mathrm{NMR}\left(100 \mathrm{MHz}, \mathrm{CDCl}_{3}+\mathrm{DMSO}-\right.$ $\left.d_{6}\right): \delta_{\mathrm{C}} 14.6,23.7,61.4,126.7,126.9,127.5,128.3,128.8$, $129.3,130.1,132.1,132.7,134.6,137.8,139.5,141.0,152.6$, $196.3 \mathrm{ppm}$; IR (KBr): $\nu_{\max } 1087,1100,1280,1434,1510$, 
1769, 2862, 2932, $3084 \mathrm{~cm}^{-1}$; MS $\left(\mathrm{ES}^{+}\right)$for $\mathrm{C}_{20} \mathrm{H}_{18} \mathrm{~N}_{2} \mathrm{O}_{2}$ 318.1 found $319.0(\mathrm{M}+\mathrm{H})^{+}, 341.0(\mathrm{M}+\mathrm{Na})^{+}$; CHN calcd. for $\mathrm{C}_{20} \mathrm{H}_{18} \mathrm{~N}_{2} \mathrm{O}_{2} \mathrm{C}, 75.45 ; \mathrm{H}, 5.70 ; \mathrm{N}, 8.80$ found C, 75.57; $\mathrm{H}, 5.69 ; \mathrm{N}, 8.82$.

1-(3-methyl-5,6-diphenylpyridazin-4-yl)ethanone (6f, Table 3). White solid; mp $132-134{ }^{\circ} \mathrm{C} ;{ }^{1} \mathrm{H}$ NMR $\left(400 \mathrm{MHz}, \mathrm{CDCl}_{3}\right)$ : $\delta_{\mathrm{H}} 1.82\left(\mathrm{~s}, 3 \mathrm{H}, \mathrm{CH}_{3}\right), 2.59\left(\mathrm{~s}, 3 \mathrm{H}, \mathrm{CH}_{3}\right), 7.18-7.47(\mathrm{~m}, 10 \mathrm{H}$, $\mathrm{Ar}-\mathrm{H}) \mathrm{ppm} ;{ }^{13} \mathrm{C} \mathrm{NMR}\left(100 \mathrm{MHz}, \mathrm{CDCl}_{3}\right): \delta_{\mathrm{C}} 21.9,27.6$, $126.7,126.8,128.5,129.3,129.7,130.0,134.6,135.8,136.6$, $138.4,138.8,151.2,152.3,186.9$ ppm; IR (KBr): $\nu_{\max } 1201$, $1240,1433,1520,1692,2888,2932,3100 \mathrm{~cm}^{-1}$; $\mathrm{MS}_{\left(\mathrm{ES}^{+}\right) \text {for }}$ $\mathrm{C}_{19} \mathrm{H}_{16} \mathrm{~N}_{2} \mathrm{O} 288.1$ found $289.0(\mathrm{M}+\mathrm{H})^{+}, 311.0(\mathrm{M}+\mathrm{Na})^{+}$; CHN calcd. for $\mathrm{C}_{19} \mathrm{H}_{16} \mathrm{~N}_{2} \mathrm{O} \mathrm{C}, 79.14 ; \mathrm{H}, 5.59 ; \mathrm{N}, 9.72$ found C, 79.33; H, 5.51; N, 9.77.

\section{Acknowledgments}

H. Mecadon thanks the University Grants Commission (UGC), India for the Financial Assistance under the RGNF scheme, and the SAIF, NEHU for the data analyses.

\section{References}

[1] M. Asif and A. Singh, "Exploring potential, synthetic methods and general chemistry of pyridazine and pyridazinone: a brief introduction," International Journal of Chem Tech Research, vol. 2, no. 2, pp. 1112-1128, 2010.

[2] A. Katrusiak, A. Katrusiak, and S. Bałoniak, "Reactivity of 6-chloro-4- and 5-hydrazino-2-phenyl-3(2H)-pyridazinones with Vilsmeier reagent," Tetrahedron, vol. 50, no. 45, pp. 12933-12940, 1994.

[3] M. P. Giovannoni, C. Vergelli, C. Ghelardini, N. Galeotti, A. Bartolini, and V. Dal Piaz, "[(3-Chlorophenyl)piperazinylpropyl]pyr-id-az-i-no-nes and analogues as potent antinociceptive agents," Journal of Medicinal Chemistry, vol. 46, no. 6, pp. 1055-1059, 2003.

[4] V. K. Chintakunta, V. Akella, M. S. Vedula et al., "3-OSubstituted benzyl pyridazinone derivatives as COX inhibitors," European Journal of Medicinal Chemistry, vol. 37, no. 4, pp. 339-347, 2002.

[5] I. G. Rathish, K. Javed, S. Bano, S. Ahmad, M. S. Alam, and K. K. Pillai, "Synthesis and blood glucose lowering effect of novel pyridazinone substituted benzenesulfonylurea derivatives," European Journal of Medicinal Chemistry, vol. 44, no. 6, pp. 2673-2678, 2009.

[6] R. Barbaro, L. Betti, M. Botta et al., "Synthesis, biological evaluation, and pharmacophore generation of new pyridazinone derivatives with affinity toward $\alpha$ - and $\alpha$-adrenoceptors," Journal of Medicinal Chemistry, vol. 44, no. 13, pp. 2118-2132, 2001.

[7] V. D. Piaz, G. Ciciani, and M. P. Giovannoni, "5-Acetyl-2methyl-4-nitro-6-phenyl-3(2H)-pyridazinone: versatile precursor to hetero-condensed pyridazinones," Synthesis, no. 7, pp. 669-671, 1994.

[8] M. F. Braña, M. Cacho, M. L. García et al., "Pyrazolo[3,4c]pyridazines as novel and selective inhibitors of cyclindependent kinases," Journal of Medicinal Chemistry, vol. 48, no. 22, pp. 6843-6854, 2005.

[9] A. A. Siddiqui, S. R. Ahamad, M. S. Mir, S. A. Hussain, M. Raish, and R. Kaur, "Synthesis and in-vitro antifungalactivity of 6-substituted-phenyl-2- [(4'-substituted phe- nyl-5' -thioxo)-1,2,4-triazol-3-yl]-methyl-2,3, 4,5-tetrahydropyridazin-3-one derivatives," Acta Poloniae PharmaceuticaDrug Research, vol. 65, no. 2, pp. 223-228, 2008.

[10] E. Sotelo, N. Fraiz, M. Yáez et al., "Pyridazines. Part XXIX: synthesis and platelet aggregation inhibition activity of 5substituted-6-phenyl-3(2H)-pyridazinones. Novel aspects of their biological actions," Bioorganic and Medicinal Chemistry, vol. 10, no. 9, pp. 2873-2882, 2002.

[11] G. A. Marriner, S. A. Garner, H. Y. Jang, and M. J. Krische, "Metallo-aldehyde enolates via enal hydrogenation: catalytic cross aldolization with glyoxal partners as applied to the synthesis of 3,5-disubstituted pyridazines," Journal of Organic Chemistry, vol. 69, no. 4, pp. 1380-1382, 2004.

[12] K. A. Ismail, A. A. El-Tombary, O. M. AboulWafa, A. M. M. E. Omar, and S. H. El-Rewini, "Novel steroidal 1,4-diketones and pyridazine derivatives as potential antiestrogens," Archiv der Pharmazie, vol. 329, no. 10, pp. 433-437, 1996.

[13] J. D. Albright, F. J. McEvoy, and D. B. Moran, "The use of $\alpha$ (aryl)-4-morpholineacetonitriles (masked acyl anion equivalents) in 1,4-additions to $\alpha, \beta$-unsaturated esters and nitriles. A verasatile synthetic route to 6-aryl-3(2H)pyridazinones," Journal of Heterocyclic Chemistry, vol. 15, p. 881, 1978.

[14] N. S. Ibrahim, F. M. A. Galil, R. M. Abdel-Motaleb, and M. H. Elnagdi, "Nitriles in heterocyclic synthesis: novel synthesis of pyridazine derivatives," Heterocycles, vol. 24, no. 5, pp. 1219$1222,1986$.

[15] G. Heinisch, W. Holzer, and G. A. M. Nawwar, "Pyridazines. XXVI. A novel synthesis of pyrano[2,3-d]pyridazines," Journal of Heterocyclic Chemistry, vol. 23, no. 1, pp. 93-96, 1986.

[16] S. Plescia, G. Diadone, J. Fabra, and V. Sprio, "Studies on the synthesis of heterocyclic compounds. Part V. A novel synthesis of some pyridazine-4- $(1 H)$ one derivatives and their reaction with hydrazine," Journal of Heterocyclic Chemistry, vol. 18, p. 333, 1981.

[17] G. E. H. Elgemeie, H. A. Elfahham, S. Elgamal, and M. H. Elnagdi, "Activated nitriles in heterocyclic synthesis: novel synthesis of pyridazines, pyridines, pyrazoles and polyfunctionally substituted benzene derivatives," Heterocycles, vol. 23, p. $1999,1985$.

[18] N. M. Abed, E. A. A. Hafez, I. Elsakka, and M. H. Elnagdi, "Activated nitriles in heterocyclic synthesis: the reaction of cinnamonitrile derivatives with active methylene reagents," Journal of Heterocyclic Chemistry, vol. 21, p. 1261, 1984.

[19] P. Schmidt and J. Druey, "Heilmittelchemische studien in der heterocyclischen reihe. Pyridazine II. Eine neue pyridazinsynthese," Helvetica Chimica Acta, vol. 37, p. 134, 1954.

[20] S. Evans and E. E. Schweizer, "A facile and general pyridazine synthesis from $\alpha$-diketone monohydrazones and $\beta$-keto esters or $\beta$-diketones," Journal of Organic Chemistry, vol. 42, no. 13, pp. 2321-2324, 1977.

[21] R. L. Nongkhlaw, R. Nongrum, and B. Myrboh, "A novel onepot synthesis of substituted pyridazines: a general method of preparation of 3,4,6-tri aryl pyridazines," Heterocyclic Communications, vol. 9, no. 5, pp. 465-472, 2003.

[22] F. M. Abdelrazek, A. M. Salah El-Din, and A. E. Mekky, "The reaction of ethyl benzoylacetate with malononitrile: a novel synthesis of some pyridazine, pyridazino[2,3-a]quinazoline and pyrrole derivatives," Tetrahedron, vol. 57, no. 9, pp. 18131817, 2001.

[23] K. Noiroj, P. Intarapong, A. Luengnaruemitchai, and S. Jai-In, "A comparative study of $\mathrm{KOH} / \mathrm{Al}_{2} \mathrm{O}_{3}$ and $\mathrm{KOH} / \mathrm{NaY}$ catalysts for biodiesel production via transesterification from palm oil," Renewable Energy, vol. 34, no. 4, pp. 1145-1150, 2009. 
[24] J. Jitputti, B. Kitiyanan, P. Rangsunvigit, K. Bunyakiat, L. Attanatho, and P. Jenvanitpanjakul, "Transesterification of crude palm kernel oil and crude coconut oil by different solid catalysts," Chemical Engineering Journal, vol. 116, no. 1, pp. 61-66, 2006.

[25] O. Ilgen and A. N. Akin, "Development of alumina supported alkaline catalysts used for biodiesel production," Turkish Journal of Chemistry, vol. 33, no. 2, pp. 281-287, 2009.

[26] J. Cléophax, M. Liagre, A. Loupy, and A. Petit, "Application of focused microwaves to the scale-up of solvent-free organic reactions," Organic Process Research and Development, vol. 4, no. 6, pp. 498-504, 2000.

[27] J. Castells and G. A. Fletcher, "The hydrolysis of 3:5-dinitrobenzoates," Journal of the Chemical Society, p. 3245, 1956.

[28] K. Sukata, "Selective $\alpha$-monoalkylation of phenylacetonitrile using alkali metal hydroxide impregnated on alumina," Bulletin of the Chemical Society of Japan, vol. 56, no. 11, pp. 33063307, 1983.

[29] K. Sukata, "The selective N-monoalkylation of amides with alkyl halides in the presence of alumina and $\mathrm{KOH}$," Bulletin of the Chemical Society of Japan, vol. 58, p. 838, 1985.

[30] E. R. H. Jones, H. H. Lee, and M. C. Whiting, "699. Researches on acetylenic compounds. Part LXIV. The preparation of conjugated octa- and deca-acetylenic compounds," Journal of the Chemical Society (Resumed), pp. 3483-3489, 1960.

[31] H. Kabashima, H. Tsuji, T. Shibuya, and H. Hattori, "Michael addition of nitromethane to $\alpha, \beta$-unsaturated carbonyl compounds over solid base catalysts," Journal of Molecular Catalysis $A$, vol. 155, no. 1-2, pp. 23-29, 2000.

[32] H. Kabashima and H. Hattori, "Cyanoethylation of methanol catalyzed by alkaline earth oxides and alumina-supported $\mathrm{K}$ catalysts," Applied Catalysis A, vol. 161, no. 1-2, pp. L33-L35, 1997.

[33] R. Neumann and Y. Sasson, "Gas phase base-catalyzed dehydrogenations of cyclic hydrocarbons over a $\mathrm{KOH} / \mathrm{Al}_{2} \mathrm{O}_{3}$ catalyst," Journal of Molecular Catalysis, vol. 35, no. 1, pp. 131136, 1986.

[34] P. Mizar and B. Myrboh, "Three-component synthesis of 5:6 and 6:6 fused pyrimidines using KF-alumina as a catalyst," Tetrahedron Letters, vol. 49, no. 36, pp. 5283-5285, 2008.

[35] P. Mizar and B. Myrboh, "Synthetic studies on KFalumina-catalysed reaction of substituted and unsubstituted aryl-oxoketene dithioacetals and $1 H$-pyrazone-5 $(4 H)$ one: a convenient synthesis of pyrazolo $[3,4-b]$ pyridine and pyrazolo $[1,5-\alpha]$ pyrimidine," Tetrahedron Letters, vol. 50, no. 25, pp. 3088-3091, 2009.

[36] M. R. Rohman and B. Myrboh, "KF-alumina-mediated Bargellini reaction,” Tetrahedron Letters, vol. 51, no. 36, pp. 4772-4775, 2010. 


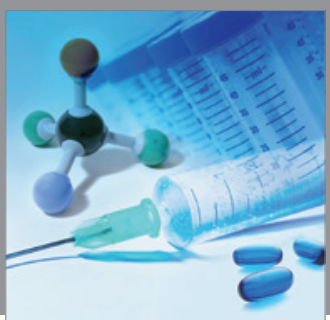

International Journal of

Medicinal Chemistry

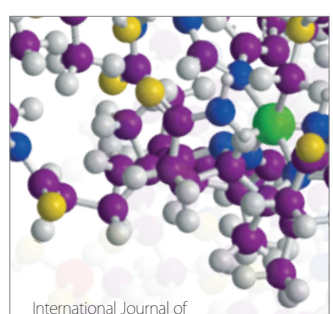

Carbohydrate Chemistry

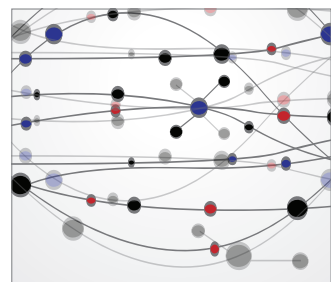

The Scientific World Journal
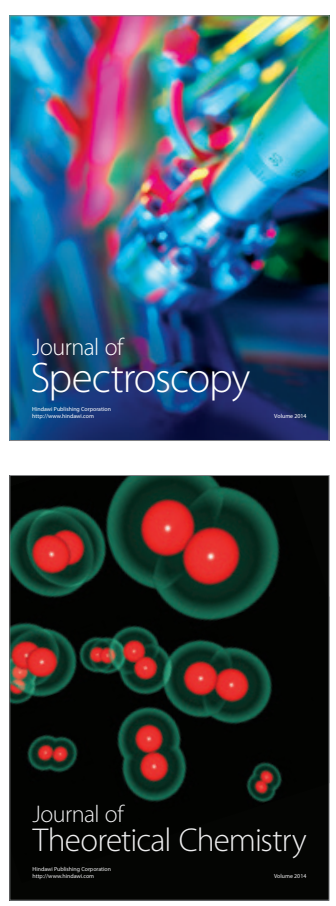
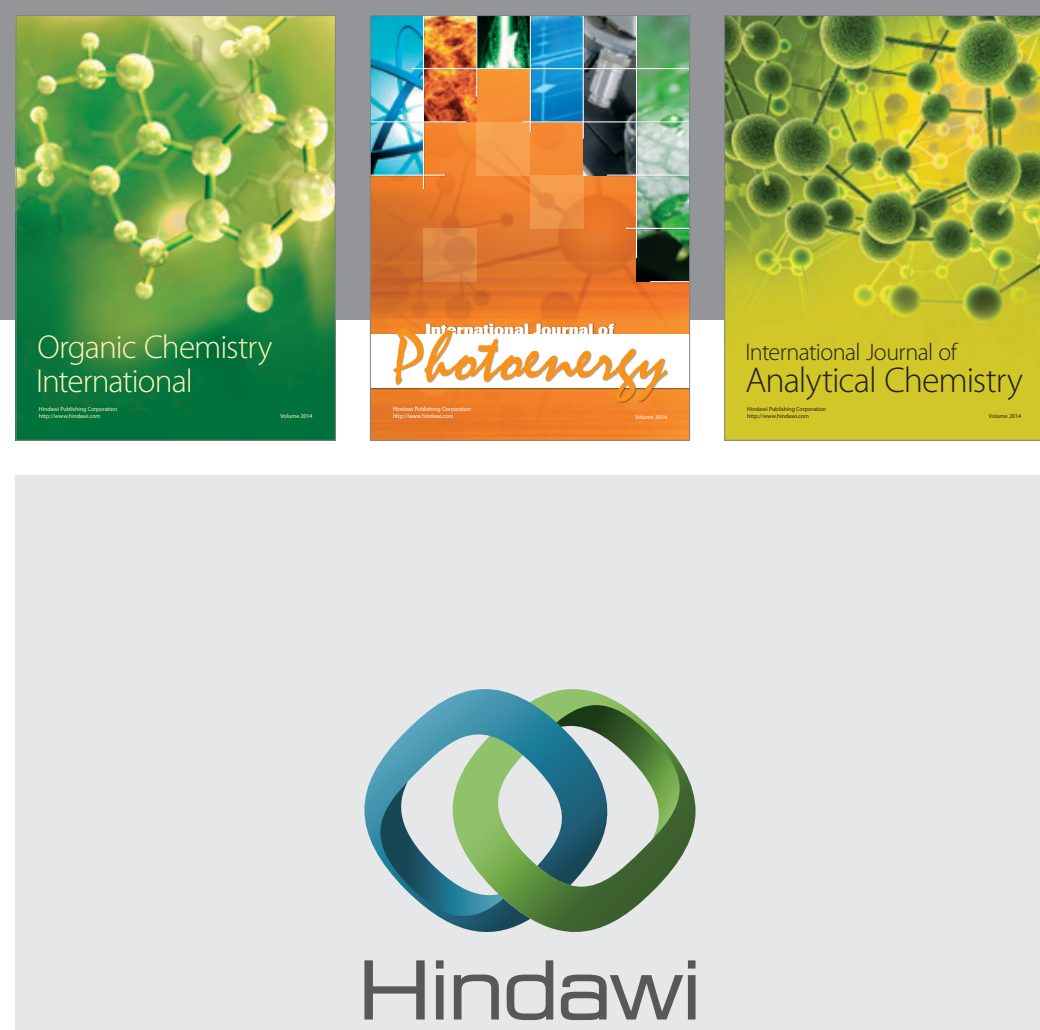

Submit your manuscripts at

http://www.hindawi.com
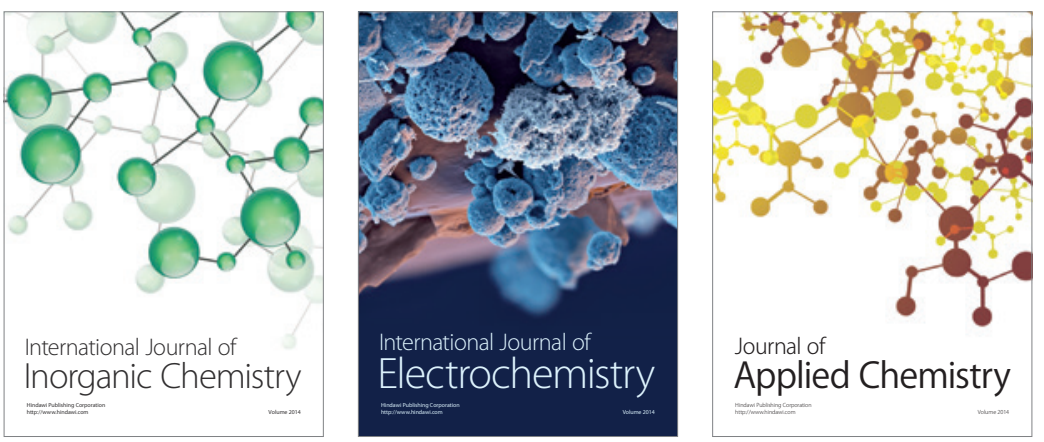

Journal of

Applied Chemistry
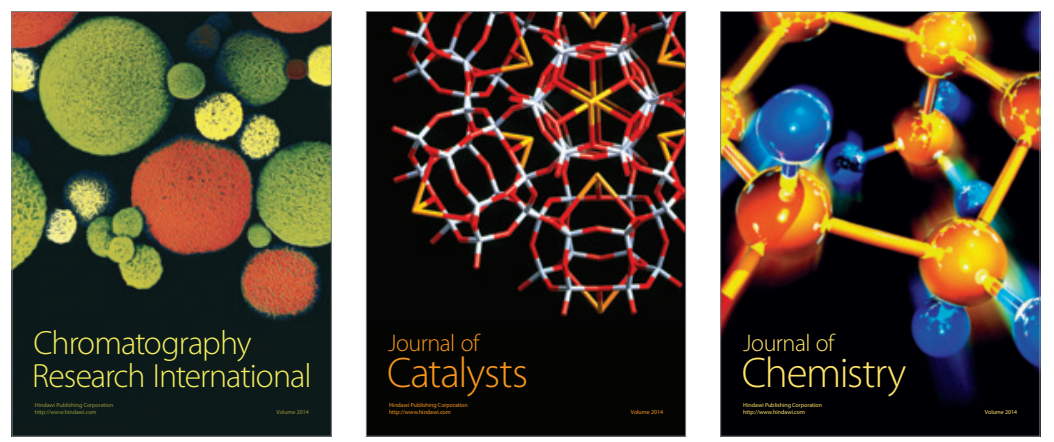
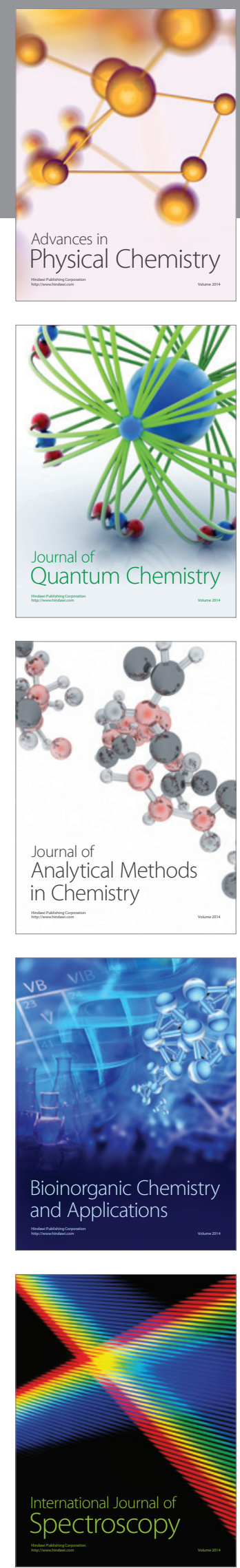